\title{
Note on Frequency Spectra of Simple Solids from Specific Heat Data
}

\author{
E. KaTz \\ Physics Department, University of Michigan, Ann Arbor, Michigan
}

(Received December 18, 1950)

\begin{abstract}
If the specific heat $C(T)$ of a solid is given as a function of temperature from $T=0$ to $\infty$ with infinite accuracy, the frequency spectrum $f(\nu)$ is uniquely determined. What information about $f(v)$ can be derived from specific heat data of experimental accuracy? The following conclusions give the answer.

(1) Experimental specific heats determine accurately the low frequency part of the frequency spectrum but allow a latitude, wide enought to fit almost any theory, for its high frequency part.

(2) In a Debye plot (effective Debye temperature $\theta$ versus $T$ ) peaks and dips in the region $0<T<\theta / 10$ represent dips and peaks in the low frequency part of the frequency spectrum. The correspondence is so simple that it can be interpreted at a glance. The
\end{abstract}

peaks and dips are superimposed on a simple Debye spectrum and presumably have a direct physical meaning, in terms of lattice irregularities. Only their centers and total weights are obtainable, so that they act effectively as single Einstein frequencies.

(3) In the region $\theta / 5<T<\infty$ all information obtainable about $f(\nu)$ consists of the first few even moments (three for specific heat errors of order one percent, five for 0.1 percent). These can be represented respectively by two or three weighted equivalent Einstein functions without direct physical meaning.

(4) The region $\theta / 10<T<\theta / 5$ corresponds to the high frequency part of the frequency spectrum, but hardly any information can be derived here.

\section{INTRODUCTION}

$S^{\mathrm{E}}$ EVERAL authors ${ }^{1}$ have attempted to refine. the Debye-Born-von Karman theory of the specific heat of solids by deriving expressions for the frequency spectrum $f(\nu)$ on the basis of more or less detailed assumptions about the elastic interactions between the atoms of the solid. The resulting specific heat*

$$
C(T)=\int^{\nu_{\max }} E(h \nu / k T) f(\nu) d \nu
$$

is then compared with experiment. Here $E(x)=(x / 2)^{2} /$ $\sinh ^{2}(x / 2)$, the well-known Einstein function. The agreement between theory and experiment is reasonable but not excellent.

The present paper centers about the inverse problem, of finding $f(\nu)$ when the specific heat $C(T)$ is given from $T=0$ to $\infty$. This is a special case of the more general problem of finding kinetic data from thermodynamic ones, treated by Bauer ${ }^{2}$ and by Blade and Kimball. ${ }^{3}$ These authors have shown that the state sum $Z(T)$ of a system determines uniquely the distribution of energy levels $g(E)$. It follows from their work that $C(T)$ determines $f(\nu)$ uniquely, since $C(T)$ obviously determines $Z(T)$ and $g(E)$ determines $f(\nu)$ uniquely. A direct method for finding $f(\nu)$ from $C(T)$ was first worked out by Montroll ${ }^{1}$ and has recently been simplified by Grayson-Smith and Stanley. ${ }^{4}$ The results were disappointing because small uncertainties in the specific

${ }^{1}$ M. Blackman, Rept. on Progress in Physics, Phys. Soc. London 8, 11 (1941). E. W. Montroll, J. Chem. Phys. 10, 218 (1942), and 11, 481 (1943). W. V. Houston, Revs. Modern Phys. 20, 161 (1948). R. B. Leighton, Revs. Modern Phys. 20, 165 (1948).

* We abbreviate $C(T)$ for the specific heat divided by its high temperature value. The present paper deals only with the constant volume specific heat due to lattice vibrations, exclusive of the electronic contribution.

${ }^{2}$ S. H. Bauer, J. Chem. Phys. 6, 403 (1938), and 7, 1097 (1939).

${ }^{3}$ E. Blade and G. E. Kimball, J. Chem. Phys. 18, 626 (1950).

${ }^{4}$ H. Grayson-Smith and J. P. Stanley, J. Chem. Phys. 18, 236 (1950). heat appear as tremendous uncertainties in the frequency spectrum. Bauer, and Blade and Kimball, reported similar large uncertainties in $g(E)$ as a result of small errors in $Z(T)$.

This state of affairs is discomforting to those who believe in the essential correctness of the theoretical frequency spectra of Blackman, Montroll, Houston, and Leighton. For, the small remaining discrepancies in the specific heat between theory and experiment may possibly mean serious errors in the frequency spectra employed by these authors.

An additional difficulty stems from the question of the constancy of the frequency spectrum with temperature. Whereas for low temperatures it is reasonable to approximate the lattice vibrations as harmonic, the anharmonicity, even when volume expansion is suppressed, must influence the result at higher temperatures. In first approximation this influence can be described as a change of the frequency spectrum with temperature, associated with a change in elastic constants. The present discussion is limited to those cases where this dependence of the frequency spectrum on temperature can be ignored. In fact, if such a dependence is present, it must be given by some other, independent source of information. It cannot be detected from specific heat data alone, since there is a one-to-one correspondence between infinitely accurate specific heat data over the entire temperature range and a-possibly fictitious-frequency spectrum satisfying Eq. (1).

Assuming a frequency spectrum which is independent of temperature, the present paper aims at revealing what details of the frequency spectrum are relevant for specific heat data of reasonable accuracy. The guiding idea throughout the present analysis is, that the Debye theory is a good first order approximation, i.e., the effective Debye temperature $\theta$, plotted versus the temperature $T$, differs only by amounts of the order of 
10 percent from a constant. $\dagger$ The deviations of $\theta$ from a constant must yield the deviations of $f(v)$ from a pure Debye spectrum. It appears expedient to divide the discussion into three parts referring to:
a. a low temperature range $0<T<\theta / 10$,
b. a high temperature range $\theta / 5<T$,
c. an intermediate range $\theta / 10<T<\theta / 5$.

\section{THE LOW TEMPERATURE RANGE $0<T<\theta / 10$}

It will be shown that all essential details of the low frequency part of the frequency spectrum can be obtained from this range.

For sufficiently low temperatures the specific heat is $\sim T^{3}$, and can therefore always be represented by $p^{3} D\left(p \theta_{0} / T\right)$, where $p$ is an arbitrary constant and $\theta_{0}$ is the value of $\theta$ extrapolated to absolute zero. The physical basis for this relation is that the low frequency vibrations, which are the only ones to be excited at low temperatures, have too long wavelengths to be influenced by the individual atoms. They must then follow the theory of vibrations in an anisotropic continuous medium, which is just the Debye theory, giving $f(\nu) \sim \nu^{2}$ and $C \sim T^{3}$.

In order to describe $C$ for somewhat higher temperatures we investigate the sum of a Debye spectrum, adapted to the very low temperature behavior of $C$, and several Einstein frequencies: ${ }^{5}$

$$
C(T)=a D\left(p \theta_{0} / T\right)+\sum_{i} \beta_{i} E\left(q_{i} \theta_{0} / T\right) .
$$

Here, the $q_{i}$ are arbitrary constants, usually less than two, indicating the frequencies. The $\beta_{i}$ are small, positive or negative weight factors for each frequency. In order to make things right at very high (see below) and at very low temperatures we require:

$$
\begin{aligned}
a+\sum_{i} \beta_{i} & =1, \\
a & =p^{3} .
\end{aligned}
$$

Introducing the effective Debye temperature $\theta(T)$ and approximating the Debye function by

$$
D(y)=4 \pi^{4} / 5 y^{3} \text {, }
$$

we get for the relative deviation $\delta=\left(\theta-\theta_{0}\right) / \theta_{0}$ the following expression:

$$
\delta=\frac{-5}{12 \pi^{4}} \sum_{i} \frac{\beta_{i}}{q_{i}{ }^{3}} \frac{x_{i}{ }^{5} e^{x_{i}}}{\left(e^{x_{i}}-1\right)^{2}},
$$

where $x_{i}=q_{i} \theta_{0} / T$.

$\dagger$ The effective Debye temperature $\theta$ is commonly defined by an implicite equation of the Debye function $D$ :

$$
C(T)=D(\theta / T) \equiv 3 \int_{0}^{1} x^{2} E(x \theta / T) d x .
$$

- A combination of Debye and Einstein spectra was previously introduced by Nernst (see E. Schroedinger, Handbuch der Physik (1926), Vol. X, p. 314). His viewpoint, however, was entirely different. His Einstein components represented optical branches for polyatomic crystals and would not occur in monatomic lattices. In the present paper this form is chosen as a way of describing mathematically any empirical $C(T)$ in a rapidly convergent series.

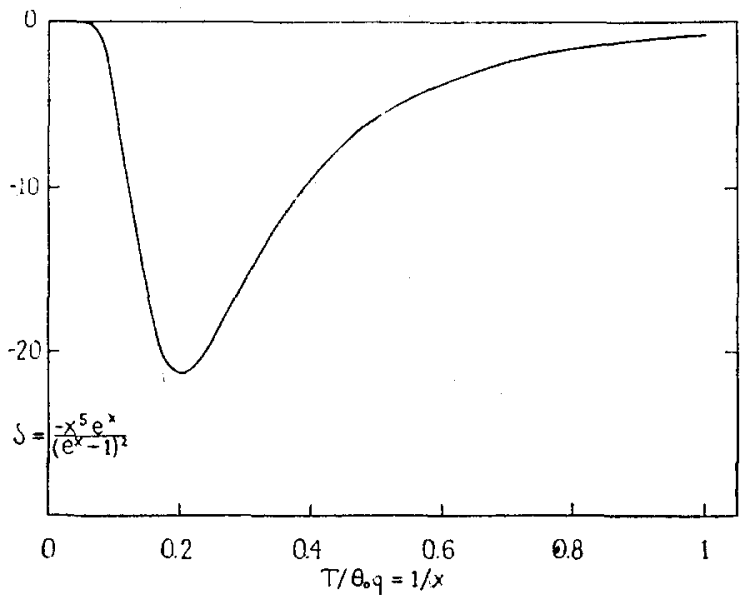

FIG. 1. The characteristic shape of the dip $\delta$ in the Debye plot for an added monochromatic frequency.

The following conclusions can be drawn from Eq. (4):

(a) $\delta$ is simply the superposition of the effects of the single frequencies.

(b) Each frequency gives rise to a dip (or, if $\beta_{i}$ is negative, a peak) in $\delta$ as a function of $T / \theta_{0}$. The dips (and peaks) all have the same basic shape, represented in Fig. 1. The shape is modified by a horizontal scaling factor depending only on $q_{i}$ and a vertical scaling factor proportional to $\beta_{i} / q_{i}{ }^{3}$.

(c) Each dip has a steep front at the low temperature side and a longer tail. Its minimum lies at $T_{m} / \theta_{0}=q / 4.93$ or $q / 5$ for all practical purposes. The depth of the minimum is $\delta_{m}=-0.09 \beta_{i} / q_{i}{ }^{3}$ $=-7.310^{-4} \beta_{i} \theta_{0}{ }^{3} / T_{m}{ }^{3}$. The frequency causing the dip is directly proportional to $T_{m}$; in fact, $\nu=5 k T_{m} / h$.

(d) Higher frequencies cause considerably smaller dips than lower ones with the same $\beta$-value (spectral weight), owing to the factor $q^{3}$. This fact may lead to important future applications of specific heat measurements at very low temperatures for the investigation of the (average) spacing of lattice perturbations in the range of 10 to 1000 lattice spacings, such as may be due to various types of hardening, mosaic structure, or impurities, etc. Superstructures of these types would give rise to slight irregularities in $f(v)$ for wavelengths of the order of their average spacing which would appear magnified in a Debye plot.

In analyzing an empirical $\theta(T)$ curve one first defines $\theta_{0}$. In view of the extreme sensitivity of the curve, near absolute zero, for the slightest deviations from a regular quadratic frequency spectrum, the value of $\theta_{0}$ may be slightly ambiguous. Such ambiguity is of a harmless nature, fortunately, as will be shown below. Next the deviations $\delta$ are analyzed in terms of dips (or peaks) of the shape as in Fig. 1, noting especially the location and depth (height) of each extremum. Thus, the frequency $q_{i}$ and the spectral weight $\beta_{i}$ of each line is obtained. The results have to be superimposed on a regular quadratic frequency spectrum corresponding to $\theta_{0}$.

The above procedure can be applied up to $T \approx \theta_{0} / 10$ or $\theta_{0} / 12$, where the error in Eq. (3) becomes 3 percent or 1 percent, respectively. The complications, arising at higher temperatures, are described below in the section of the intermediate range. Inspection of experimental curves such as in Fig. 2 indicates that not many 


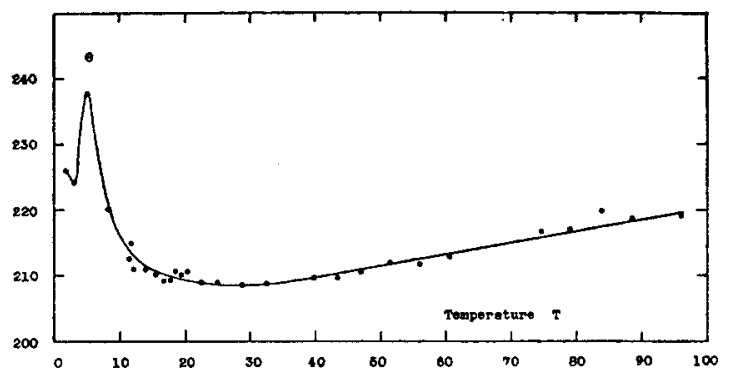

FIG. 2. Experimental specific heat for silver from literature (see R. B. Leighton, reference 1).

more than four dips or peaks can be analyzed from each curve in the low temperature range. The data will not allow, in general, drawing any conclusion concerning the shape of the corresponding peak in $f(\nu)$ but give only its average location and total weight. Hence, this represents all the significant detail obtainable in this region.

The following figures give an idea of the sensitivity of the method: at $q=0.1$ (frequency 0.1 of the fictitious maximum frequency of the quadratic spectrum with the same $\theta_{0}$ ) and $\beta=10^{-3}$, one has $\delta_{m}=0.09$; for $q=0.5$ (the limit of the low $T$ range) and $\beta=0.014$, one has $\delta_{m}=0.01$.

We return now the the question of what happens if $\theta_{0}$ is off by a relative amount $d_{0} \ll 1$. It is easily shown that this changes $f(v)$ by adding a little bit of a quadratic Debye spectrum to it. Quantitatively, a spectrum $\varphi(\nu) d \nu=-9 d_{0}\left(h / k \theta_{0}\right)^{3} \nu^{2} d \nu$ is added between $\nu=0$ and $k \theta_{0} / 2 h$. It is clear that this spectrum will never introduce spurious peaks, nor affect the recognition of local maxima or minima, and is thus rather harmless.

\section{THE HIGH TEMPERATURE RANGE $T$ \% $\theta / 5$}

Assuming a frequency spectrum which is independent of temperature, the following discussion shows that the high temperature specific heat data yield at most information equivalent to the first three even moments of the frequency spectrum.

We replace the frequency spectrum by a substitute spectrum giving within the limits of error the same specific heat. The substitute spectrum ${ }^{6}$ consists of $s$ weighted single frequencies $\nu_{1} \cdots \nu_{s}$ with weights $\alpha_{1} \cdots \alpha_{s}$, where $\sum_{j} \alpha_{j}=1$. This introduces $2 s-1$ parameters and we have

$$
C(T) \approx \sum_{j} \alpha_{j} E\left(h \nu_{j} / k T\right) .
$$

The question is now: How many frequencies must be used for a reasonable approximation of $C(T)$ or $\theta(T)$ ? In answering this question we expand all quantities involved in inverse powers of $T^{2}$.

Suppose an empirical function $\theta(T)$ is given. One will

- The qualitative merit of this substitute spectrum was previously pointed out by E. W. Montroll, Quart. App. Math. 5, 223 (1947). then determine the constants $p_{i}$ of the expansion:

$$
\left[\theta(T) / \theta_{\infty}\right]^{2}=1+\sum_{i} p_{i}\left(\theta_{\infty} / T\right)^{2 i},
$$

where $\theta_{\infty}$ is the constant value, approached by $\theta$, for temperaturea above $\theta_{\infty}$. On account of the weak dependence of $\theta$ on $T$ the constants $p_{i}$ must be small, and would vanish for an ideal Debye spectrum. On the other hand, the Debye temperature $\theta(T)$ is defined by that value of $y=\theta / T$ which will make the Debye function $D(y)$ equal to $C(T)$ :

$$
C(T)=D(y) \equiv 1+\sum_{n=1}^{\infty} b_{n} y^{2 n},
$$

where

$$
b_{n}=(-)^{n} 3(2 n-1) B_{n} /(2 n) !(2 n+3)
$$

and $B_{n}$ are the Bernoulli numbers $(1 / 6 ; 1 / 30 ; 1 / 42$; $1 / 30 ; 5 / 66 ; \cdots)$. This expansion converges rapidly for $y<2 \pi$, the radius of convergence. Substituting Eq. (6) into Eq. (7), one obtains an expansion of $C$ in inverse powers of $T^{2}$ :

$$
C(T)=1+\sum_{n=1}^{\infty} c_{n}\left(\theta_{\infty} / T\right)^{2 n}
$$

where

$$
\begin{aligned}
& c_{1}=-1 / 20 \\
& c_{2}=\left(1 / 28-p_{1}\right) / 20 \\
& c_{3}=-\left(5 / 4536-p_{1} / 14+p_{2}\right) / 20 \\
& c_{4}=\left(1 / 31680-5 p_{1} / 1512+\left(p_{1}^{2}+2 p_{2}\right) / 28-p_{3}\right) / 20 \\
& \text { etc. }
\end{aligned}
$$

This has to be matched by the corresponding expansion of Eq. (5) with

$$
E(x)=1+\sum_{n=1}^{\infty} a_{n} x^{2 n}
$$

where

yielding

$$
a_{n}=(-)^{n}(2 n-1) B_{n} /(2 n) !,
$$

$$
C(T) \approx 1+\sum_{n=1}^{\infty} a_{n}(h / k T)^{2 n}\left(\sum_{j=1}^{s} \alpha_{j} \nu_{j}^{2 n}\right) .
$$

The number of terms that can be matched obviously is $2 s-1$ and the first unmatched term (number $2 s$ ) of the difference between Eqs. (10) and (8) is a measure for the remaining error in $C(T)$. In fact, because the terms of this difference have usually alternating sign, this term sets an upper limit to the absolute error $\Delta$ in $C$. Moreover, the relative error $\delta$ in $\theta$ is easily seen to be

$$
\delta=10 \Delta T^{2} / \theta_{\infty}{ }^{2}
$$

in the temperature range under discussion.

$$
\text { Case } s=1
$$

This case has one single frequency $\nu_{1}$ and is actually the old Einstein approximation. The matching of Eqs. 
(10) and (8) leads to the single condition:7

$$
\theta_{\infty}{ }^{2}=(5 / 3)\left(h \nu_{1} / k\right)^{2} \equiv(5 / 3) \theta_{E^{2}}{ }^{2}
$$

where $\theta_{E}$ is the "Einstein" temperature defined by the second part of (12). When comparisons between the theories of Einstein and Debye have been made, curves of $C$ versus $T$ for equal $\theta_{\text {Debye }}$ and $\theta_{\text {Einstein }}$ have usually been given. ${ }^{8}$ These curves seem to differ considerably, but a proper scaling factor of $(5 / 3)^{0.5}$ would bring out their equivalence over a substantial temperature range. In order to see how far this range extends, we consider the term $n=2$ for Eqs. (10)-(8), giving

$$
\Delta \approx\left(\theta_{D} / T\right)^{4}\left[(-1 / 3500)+\left(p_{1} / 20\right)\right] .
$$

For a pure Debye spectrum $p_{1}=0$ and so for all temperatures down to $\theta / 2$, the error in $C$ is less than one percent and so is $\delta$ practically. This means that, from an experimental standpoint, the Debye and Einstein approximations are fully equivalent for $\theta / 2<T<\infty$. For actual spectra $p_{1}$ is usually negative but rarely much more than 0.005 . Consequently, all spectra are equivalent with the Einstein approximation for temperatures down to $\theta / 1.5$ or $\theta / 2$. And this is all the information concerning the frequency spectrum that can be derived from specific heat data in this temperature range.

\section{Case $s=2$}

In this case the specific heat is approximated by a combination of two Einstein functions $\alpha E\left(x_{1}\right)+$ $(1-\alpha) E\left(x_{2}\right)$ with frequencies $\nu_{1}$ and $\nu_{2}$. The matching of Eqs. (8) and (10) gives the three equations:

$\alpha\left(x_{1} / y_{\infty}\right)^{2 n}+(1-\alpha)\left(x_{2} / y_{\infty}\right)^{2 n}=c_{n} / a_{n} \quad(n=1,2,3)$.

Consider first the special case of a pure Debye spectrum. The solutions of Eqs. (14) are then

$$
\begin{aligned}
\alpha & =0.5837, \\
x_{1} / y_{\infty} & =0.906, \\
x_{2} / y_{\infty} & =0.538 .
\end{aligned}
$$

In this approximation the Debye function is therefore equivalent to

$$
D(y) \approx 0.584 E\left(0.906 y_{\infty}\right)+0.416 E\left(0.538 y_{\infty}\right) .
$$

This seems to be the true reason why a similar formula by Nernst and Lindemann, ${ }^{9}$

$$
C(T) \approx 0.5 E(y)+0.5 E(0.5 y),
$$

worked quite well. From the above discussion it is seen that the reason for the validity of Eqs. (15) and (16) is

\footnotetext{
7 This relation differs slightly from that given by Mott and Jones in Properties of Metals and Alloys (Oxford University Press, 1936), p. 8 , whose factor is $16 / 9$ instead of $5 / 3$.

8 See for instance F. Seitz, Modern Theory of Solids (McGrawHill Book Company, Inc., New York, 1950), p. 104 or E. Schroedinger, reference 5 .

${ }^{9}$ Mott and Jones, reference 7, p. 8, or M. Blackman, reference 1, p. 13 .
}

not, that the frequency spectrum has two peaks at frequencies differing by a factor of approximately two. The perfectly smooth Debye spectrum already creates this feature and it cannot be used, therefore, as an argument in support of such two-maxima spectra as calculated by Blackman, et al.

In order to see over what temperature range this approximation is valid we consider the term $n=4$ for Eqs. (10)-(8), giving:

$$
\delta \approx 2.610^{-7}\left(\theta_{\infty} / T\right)^{6} .
$$

Numerically, this means $\delta=0.3$ percent for $T=\theta_{\infty} / 5$ and 1.1 percent for $T=\theta_{\infty} / 6$. Since the radius of convergence of all these series expansions lies at $T=\theta_{\infty} / 2 \pi$, this is about as far as one can go with this type of approximation for the Debye spectrum. For a general spectrum the situation is essentially the same as will now be shown.

In the general case $\left(p_{i} \neq 0\right)$ the solutions of Eqs. (14) are, fully written out:

with

$$
\begin{aligned}
\alpha & =0.5+0.0837 f \\
x_{1} / y_{\infty} & =0.906 g \\
x_{2} / y_{\infty} & =0.538 h
\end{aligned}
$$

$$
\begin{gathered}
f=\left(1-49612.5 p_{2}\right) /\left\{1-283.5\left(9 p_{1} / 5+49 p_{2} / 5\right.\right. \\
\left.\left.-315 p_{1}^{2}-1944810 p_{2}^{2} / 8+18375 p_{1}^{3}\right)\right\}^{0.5} \\
g^{2}=\left[1-130 p_{1}+2720 p_{2}+0.328(N-1)\right] /\left(1-175 p_{1}\right) \\
h^{2}=\left[1-362 p_{1}+7600 p_{2}-0.915(N-1)\right] /\left(1-175 p_{1}\right),
\end{gathered}
$$

and $N$ is the denominator of $f$ and can often be approximated by $\left(1-280 p_{1}\right)$. The expressions for $f, g, h$ show that the value of $\alpha$ never deviates far from 0.5 and the ratio $x_{1} / x_{2}$ is always about 2 , for values of $0<p_{1}<10^{-2}$ and $0<p_{2}<10^{-4}$, which more than covers the range of these variables in many trial cases.

The error term $n=4$ is given by

$$
\delta \approx 2.610^{-7}\left(\theta_{\infty} / T\right)^{6}(1+m),
$$

where $m \rightarrow 0$ when $p_{12} \rightarrow 0$. The exact expression for $m$ is too lengthy to be given here. A typical case of $p_{1}=-0.005$ and $p_{2}=2.10^{-5}, p_{3}=0$ gives $m \sim 1$, which proves the contention that Eqs. (19) and (17) are practically equivalent.

\section{Case $s=3$}

This case will be of only theoretical interest unless specific heat measurements become possible with an accuracy far better than obtainable at present. Here we have to match three weighted single frequencies:

$$
\alpha_{1} E\left(x_{1}\right)+\alpha_{2} E\left(x_{2}\right)+\left(1-\alpha_{1}-\alpha_{2}\right) E\left(x_{3}\right) .
$$

One now obtains five equations of a structure similar to Eq. (14). For a pure Debye spectrum this yields the following solutions:

$$
\begin{aligned}
& \alpha_{1}=0.350 \quad \alpha_{2}=0.461 \\
& x_{1} / y_{\infty}=0.950 \quad x_{2} / y_{\infty}=0.742 \\
& \begin{aligned}
1-\alpha_{1}-\alpha_{2} & =0.189 \\
x_{3} / y_{\infty} & =0.406 .
\end{aligned}
\end{aligned}
$$




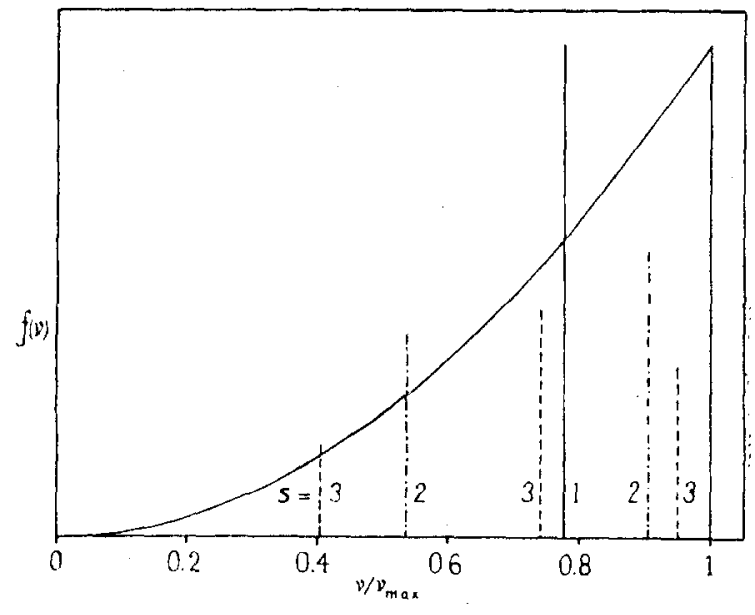

Fig. 3. High temperature analysis of Debye spectrum into 1, 2, 3 equivalent Einstein components.

The range of validity is given by the relative error $\delta$ in $\theta$ :

$$
\delta \approx 0.08\left(\theta_{\infty} / 10 T\right)^{10}
$$

which is less than 0.1 percent for $\theta_{\infty} / T=2 \pi$. A similar order of magnitude may be expected for any reasonable frequency spectrum. It is unlikely that any closer approximation will ever be needed for this temperature region. The relations for the cases $s=1,2,3$ are graphically illustrated for the Debye spectrum in Fig. 3.

\section{CONNECTION WITH MONTROLL'S MOMENTS}

The matching of the Eqs. (8) and (10) is evidently similar to giving the first $2 s-1$ moments $M_{k}$ defined by

$$
M_{k}^{2 k}=\int_{0}^{\nu_{\max }} f(\nu) \nu^{2 k} d \nu
$$

where $f(\nu)$ is normalized such that its integral is unity. However, the present method of representing the spectrum by single frequencies takes into account a little more than these moments only. It incorporates the fact that certain inequalities exist between moments, ${ }^{10}$ in particular:

and

$$
M_{k+1} \geqslant M_{k} \quad(k>0)
$$

$$
M_{k+2} / M_{k+1} \geqslant\left(M_{k+1} / M_{k}\right)^{k / k+2}
$$

or, in general:

$$
M_{k+p+q} / M_{k+p} \geqslant\left(M_{k+p} / M_{k}\right)^{q k / p(k+p+q)} \quad k, p, q>0 .
$$

Knowledge of the first $2 s-1$ moments yields lower bounds for all higher moments. This information is just incorporated by using the frequency spectrum consisting of $s$ single frequencies. Among all spectra with the same first $2 s-1$ moments this type gives to moments beyond the $(2 s-1)$ th their minimum values,

${ }^{10} \mathrm{H}$. Cramer, Mathematical Methods in Statistics (Princeton University Press, Princeton, 1946), p. 255. consistent with the above relations. This additional information concerning the higher moments as used in the present method results in errors that are almost an order of magnitude less than would be the case if the series were simply cut off after the terms containing the known moments.

The connections between the moments and the constants $p$ used here are

$$
\left(h M_{k} / k \theta_{\infty}\right)^{2 k}=c_{k} / a_{k},
$$

where the Boltzmann $k \theta_{\infty}$ will not be confused with the index $k$ in all other places of the formula.

\section{THE INTERMEDIATE RANGE $\theta / 10<T<\theta / 5$}

In this range, it will be shown, very little information about the high frequency part of the frequency spectrum can be obtained. An approach similar to that used for low temperatures will be followed, but the dependence of $D$ on $T$ is no longer simply Eq. (3). One has the well-known asymptotic expansion:

$$
\begin{gathered}
D(y)=4 \pi^{4} / 5 y^{3}-\Delta(y) \\
\Delta(y)=12 y \sum_{n=1}^{\infty} e^{-n y}\{(1 / 4)+(1 / n y) \\
\left.y=\theta / T . \quad+\left[3 /(n y)^{2}\right]+\left[6 /(n y)^{3}\right]+\left[6 /(n y)^{4}\right]\right\}
\end{gathered}
$$

In the low temperature range $\Delta$ can be neglected; in the intermediate range all terms $n>1$ may be neglected for an error of less than 1 percent. Setting $y=y_{0}(1+\delta)$, where $y_{0}=\theta_{0} / T$, we have to find $\delta$ as a function of $T$ satisfying:

$$
D(y)=p^{3} D\left(p y_{0}\right)+\sum_{i} \beta_{i} E\left(q_{i} y_{0}\right) .
$$

Substituting Eq. (20) into Eq. (21), expanding about $y_{0}$, and retaining only terms up to the first power in $\delta$ one finds:

$$
\delta=-\frac{\sum \beta_{i} E\left(q_{i} y_{0}\right)+\left\{\Delta_{0}-p^{3} \Delta\left(p y_{0}\right)\right\}}{\left(12 \pi^{4} / 5 y_{0}^{3}\right)+\Delta_{0} u_{0}},
$$

where $u=d \log \Delta / d \log y$ and the subscript zero refers to the value taken for $y=y_{0}$. In discussing Eq. (22) we note first that a term has been added to the numerator and the denominator, but otherwise this formula is similar to Eq. (4). Consider first the first term of the numerator together with the denominator. The value of $u_{0}$ in this range is almost exactly equal to $-y_{0}$. The denominator varies between $0.91\left(12 \pi^{4} / 5 y_{0}{ }^{3}\right)$ at $y_{0}=10$ and $0.31\left(12 \pi^{4} / 5 y_{0}{ }^{3}\right)$ at $y_{0}=5$. For a qualitative analysis the coefficient could be taken 0.6 throughout this range. The effect of the changing coefficient is to counteract the rapid decrease in sensitivity of $\delta$ somewhat, and to modify the shape of the dip that corresponds to a single frequency a little. Its maximum is shifted to the left, making the front steeper and the tail longer. These changes do not affect the general qualitative features of the analysis. More important are the effects arising from the added term in the numerator. 
This term, together with the denominator, represents a contribution to $\delta$, the absolute value of which rises gradually from practically zero at $y_{0}=10$ to a maximum at $y_{0} \approx 4$, and this maximum is about $0.3 \sum \beta_{i}$. Consequently, if $\sum \beta_{i} \approx 0$, then the effects of this contribution vanish; but if this sum differs markedly from zero, an effect is produced through this term which has to be compensated by adding a single frequency at this spot. However, it turns out that this gives a major contribution to $\sum \beta_{i}$, always in the sense of increasing its absolute value. This aggravates the second-term influence, etc. The question is now whether this procedure converges rapidly. Unfortunately, it is divergent or very slowly convergent and therefore cannot be used. In practice the only way to avoid this difficulty is to make sure that $\sum \beta_{i}=0$ and this can always be done by choosing $\theta_{0}$ about equal to $\theta$ at $T=\theta / 4$. (See the final remark in the low temperature section.)

With these precautions Eq. (22) can now be used up to $q \approx 1$. It is not practical to attempt extending the present method to greater values of $q$ (or $T$ ). In the first place the method loses elegance and ease of comprehension. Secondly, sensitivity is lost with higher $q$ (at $q=1$ and $\delta_{m}=0.01$ one has $\beta=0.04$, in other words, a dip with area of 4 percent of the total area of the frequency spectrum just escapes detection). Finally, the divergent or slowly convergent effects outlined above stop further extension of the method.

As a rule of thumb, one may say that the present approach divides the actual frequency spectrum into two parts, roughly in half on the frequency scale. The low frequency part can be obtained from specific heat data with reasonable accuracy. As one approaches the halfway mark, the method becomes rapidly insensitive; and for the high frequency part one sees very little detail. The halfway mark corresponds to a value of $q$ somewhere between 0.5 and 1 . The spectrum may, of course, extend beyond $q=1$, since the Debye limit is not necessarily the real limit.

\section{AN EXAMPLE OF FITTING}

The data of Fig. 2, copied from Leighton's paper, ${ }^{1}$ show the Debye temperature as a function of the temperature for silver. We take $\theta_{0}=212^{\circ}$, the value of $\theta$ near $T=\theta / 4$. The graph indicates in the low temperature region:

(1) A dip in $f(v)$ for $q=0.04$, corresponding to a maximum in $\theta(T)$ at $1.6^{\circ}$. The height of this maximum is about $13^{\circ}$ or 6 percent and so we have $\beta_{1}=-0.4 \times 10^{-4}$.

(2) A dip in $f(\nu)$ for $q=0.12$. Taking into account the tail of the first dip, the depth of this one is $23^{\circ}$ or $\delta_{m}=0.11$; and so we have $\beta_{2}=-2.1 \times 10^{-3}$

(3) A peak in $f(\nu)$ at $T=14^{\circ}$ or $q=0.33$. The maximum depth of the corresponding $\operatorname{dip}$ in $\theta$ is 3.0 percent and we have $\beta_{3}$ $=+0.012$.

This description fits the data, as shown in Fig. 4, to within the experimental error, over the entire range from 0 to $60^{\circ}$. Since $\sum \beta=0.010$, the complications of divergence can be ignored in the intermediate range.

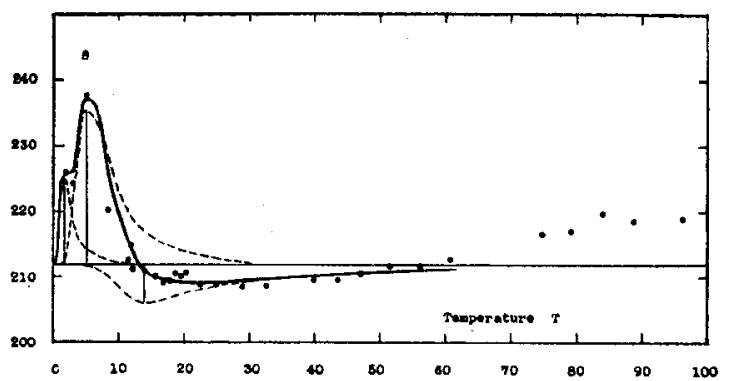

Fig. 4. Analysis of data in terms of a Debye spectrum and superimposed monochromatic frequency peaks or dips. The full drawn curve is the sum of the three dotted curves and $\theta_{0}=212^{\circ}$.

The few points at higher temperatures are not accurate enough to warrant an analysis for the high temperature range.

\section{CONCLUDING REMARKS}

It has been shown that two main types of information about the frequency spectrum can be obtained.

From the low temperature specific heat data one obtains physically real peaks and dips of approximately monochromatic character in the low frequency part of the frequency spectrum. Their detection becomes extremely sensitive at low temperatures. Insofar as they cannot be accounted for by differences between the results of lattice theories and the Debye theory, the effects of which usually do not extend much below $\theta / 10$, they may be caused by mosaic structure and other lattice imperfections. This hypothesis can, in principle, be tested by influencing the lattice structure in a known way and measuring low temperature specific heats. In the opinion of the author, these peaks and dips may be ascribed to such structure sensitive causes rather than being attributed to experimental errors in the (old) specific heat measurements, as was suggested by Casimir. ${ }^{11}$ More detailed speculations, however, seem premature until more systematic experimental data are available.

On the other hand, from high temperature specific heats one can in practice obtain at best the first three to five even moments of the frequency spectrum and some limitations concerning higher moments. This information can best be incorporated by representing the spectrum by two or three weighted "effective" Einstein frequencies which possesses, however, no direct physical meaning but may be useful parameters for the comparison of data. All theoretical predictions concerning finer details of the high frequency part of the spectrum must escape observation.

It is a pleasure to thank Professor G. E. Uhlenbeck for helpful criticism of the manuscript.

"1 H. B. G. Casimir, oral communication at the summer symposium lectures, Ann Árbor, 1948. It was suggested that Keesom's measurements on $\mathrm{KCl}$, which show a similar type of fine structure, were not accurate enough to warrant theorizing about this point. This suggestion was based on a discussion of this question between Casimir and Keesom. 